\title{
SALUD
}

\section{Utilización de bifosfonatos en el tratamiento de la displasia fibrosa de los maxilares}

\author{
Fanny Graciela Ayala Ratti ${ }^{1}$
}

\begin{abstract}
Resumen
Introducción: El objetivo de este trabajo es, el de exponer las diferentes técnicas de tratamiento para la Displasia Fibrosa de los maxilares, utilizando bibliografía actualizada.

Teniendo en cuenta que dicha dolencia es benigna y que consiste en el reemplazo progresivo del hueso normal por tejido fibroso inmaduro, poco común y de etiología desconocida, esta revisión intentará dilucidar el tratamiento más eficaz y el menos invasivo para dicha patología.

Se han detallado varias alternativas de tratamiento para el mismo; la enucleación quirúrgica y el uso de los derivados bifosfonatos para el tratamiento de dicha patología, que, si bien reducen el dolor y la velocidad de recambio óseo en la zona, conlleva a su vez una nueva complicación en la terapia: la osteonecrosis de los maxilares.

Luego de la exhaustiva revisión bibliográfica, se ha determinado la terapéutica de elección que consiste en: Evaluar detenidamente al paciente diagnosticado con una displasia fibrosa, así como la importancia de la elaboración de una correcta historia clínica, para establecer si el mismo consume bifosfonatos, reconocer las complicaciones que se derivan del uso a lo largo del tiempo y proceder a la remoción quirúrgica si así el paciente y las condiciones clínicas lo ameriten.
\end{abstract}

Objetivo: Determinar la eficacia de la utilización de Bifosfonatos en el tratamiento alternativo en la displasia fibrosa Monostotica y sus ventajas respecto a la terapéutica quirúrgica convencional

Material y Método: Este estudio se basó en una revisión bibliográfica actualizada Resultados: Como Odontólogos debemos realizar una buena historia clínica del paciente, examinar cuidadosamente la cavidad bucal buscando la presencia de exposiciones óseas, conocer que tipos de medicamentos está recibiendo el paciente si el mismo está recibiendo bifosfonatos, que los mismos son capaces de producir osteonecrosis en el maxilar.

Conclusiones: Según las bibliografías consultadas la utilización de los

1. Universidad María Auxiliadora.

Es una tesis para optar el título de Especialista en Cirugía Maxilofacial.

E-mail: fananayalar@gmail.com

DOI: 10.26885/rcei.foro.2018.116 
bifosfonatos en el tratamiento de las Displasias fibrosas monostoticas o poliostoticas, no sería el tratamiento de elección.

La prevención es la llave para limitar el desarrollo de las osteonecrosis en los maxilares, por este motivo el odontólogo debe estar muy alerta sobre la medicación de sus pacientes, para ello debe realizar una exhaustiva historia clínica, examen bucal minucioso.

Palabras clave: bifosfonatos, osteo necrosis, displasia fibrosa.

\section{Referencias}

Ceccotti, E. (2007). El diagnóstico en clínica estomatológica. México: Panamericana.

De Conto, F., Dias, F., Rhonden, R. (200). Displasia Fibrosa nonostotica de los maxilares. Revista Brasilera de Patología Oral. Recueprado de www. patologíaoral.com.br

Kumar, V., Abbas, A. K., Fausto, N. (2009). Patología estructural y funcional (7a ed.) Barcelona: Elsevier.

Casiglia, J., Epstein, J., Jacobsen, P. L., Siegel, M. A., Woo, S. B. (2005). Managing the care of patients with bisphosphonate-associated osteonecrosis. Journal American Dental Association, (136), 1658-68.

Sapp, P., Eversole, L. R., Wysocki, G. (2004). Patología oral y maxilofacial contemporánea. Barcelona: Elsevier. 